\title{
Statistical Analysis of the Quality of Date Production for 2019 in Iraq
}

\author{
Nadia A. Ayed \\ nidea.iead@uobasrah.equ.iq \\ Department of Statistics, \\ College of Administration and Economics, \\ University of Basra, Basra, Iraq
}

Received on: $17 / 05 / 2020$

Accepted on: 29/06/2020

\begin{abstract}
Dates are a great food that meets many human needs, and the government should encourage and intensify efforts to support the expansion of palm cultivation and development and focus on its production in our country as a means of food security in the future. In addition, palm trees have an important economic role in improving the country's economy. In this paper, a sharp decline in the quality of production will be highlighted. So, the aim of the study is to know the most important types of palm trees that affect the production of tigers for the year 2019 if the study includes 11 governorates, and the multiple linear regression method has been applied in selecting the most important types affecting the production.. and the types of palm trees did not appear Halawi and Khattawi and Khadari any effect on Dates production in the aforementioned year, although these types are considered to be one of the desirable first-class varieties in the Iraqi market, and for export in particular, in a manner that supports the country's economy.

Keywords: Stepwise Multiple Regressions; Estimate parameters of the model; The Coefficient of Determination; Descriptive Statistics; ANOVA.

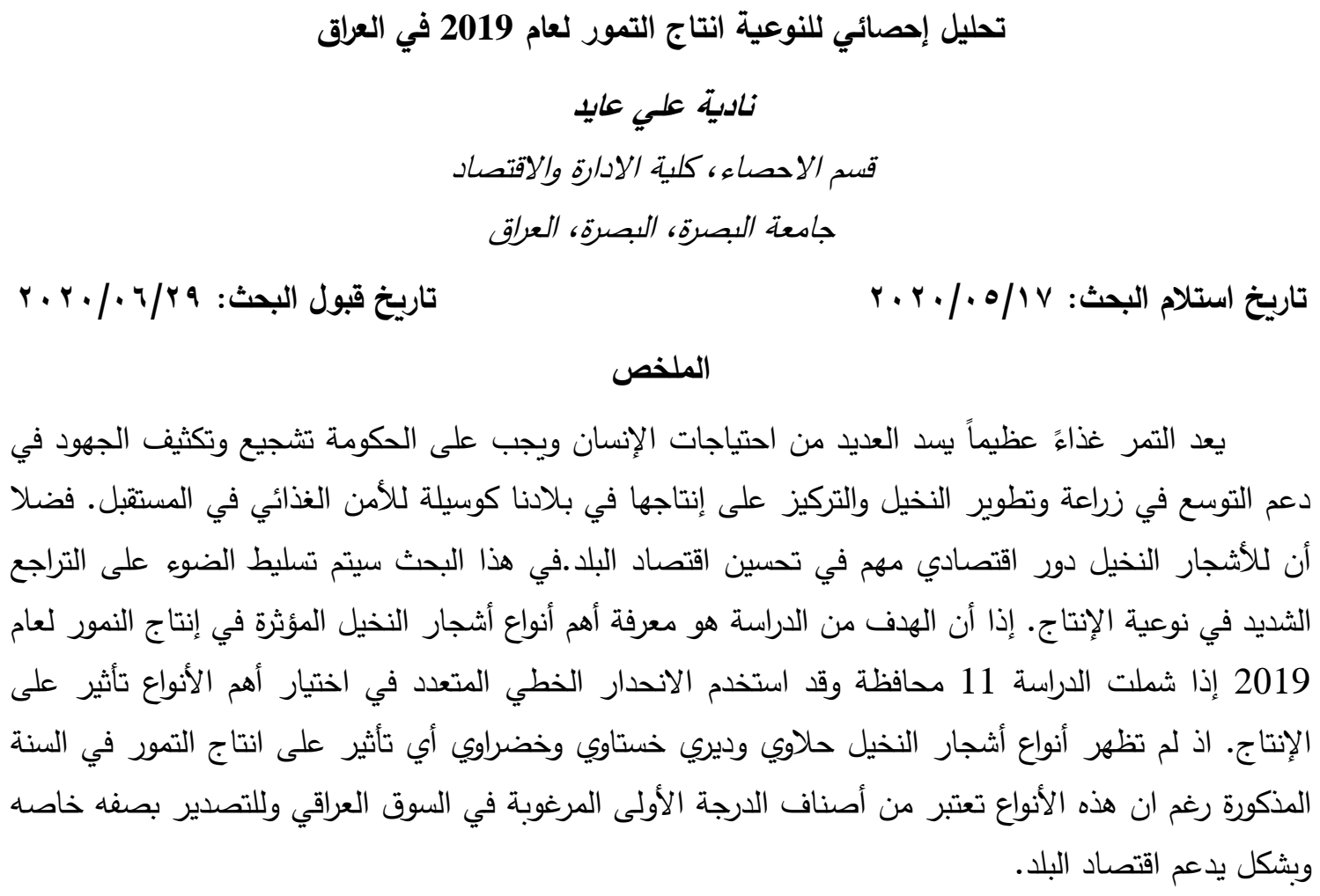


الكلمات المفتاحية: الانحدار المتعدد التدريجي، تقدير معلمات الانموذج، معامل التحديد، الاحصاءات الوصفية، تحليل التباين.

المقدمة

بعد أن تصدر العراق المرتبة الأولى عالميا من ناحية زراعة أشجار النخيل في سبعينيات القرن الماضي ألا

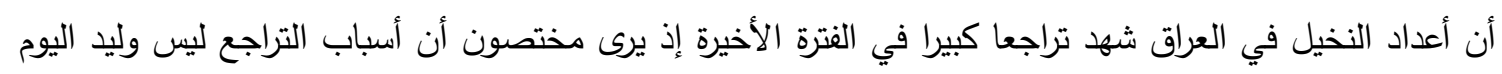

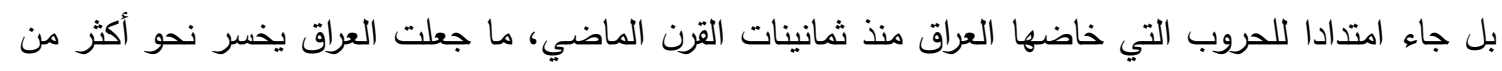
14 مليون نخلة. وتعد أشجار النخيل الثريان الرئيس للاقتصاد العراقي نظرا لما يقوم به العراق من تصدير منتجات التمور

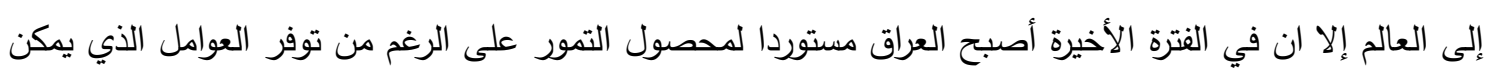

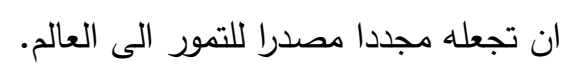
هنالك أسباب عديدة لتراجع أعداد النخيل في العراق منها الحرب العراقية الإيرانية وحرب الخليج الثانية التي

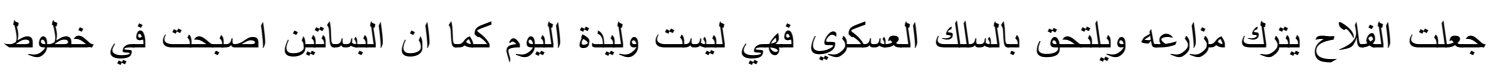

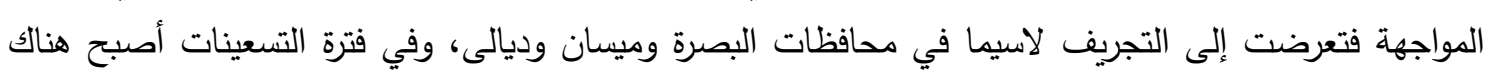

إهمال حكومي للنخيل.[1] مشكلة البحث:

في الفترة الأخيرة شهد العراق تدهور خطير في أنتاج وأعداد أشجار النخيل ومن أسباب ذلك عن تجريف الأراضي الزراعية وبيعها قطع سكنية وشحت الحصة المائية المخصصة وعدم وجود جهة حكومية تتبنى تصدير التمور نتيجة عن ذلك أن إيرادات التمور اصبحت لا تغطي كلفة نقلها فضلا عن عدم دعم الدولة للفلاح. سنحاول في هذا البحث تسليط الضوء على هذا التراجع الثديد في نوعية الانتاج. هدف البحث: يهدف البحث إلى أجراء تحليل إحصائي لإنتاج التمور لعام 2019 لغرض معرفة نوعية الإنتاج باستخدام عدد أشجار النخيل حسب الأصناف للعام نفسه.

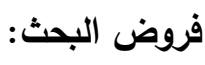
لخصت فروض البحث حول مدى معنوية تأثير المتغيرات المستقلة في نموذج الانحدار على المتغير التابع (كمية إنتاج التمر في عام 2019) المتغيرات المستقلة: - معنوية عدد أشجار نخيل زهدي

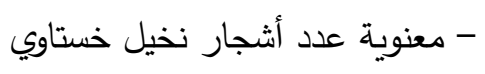

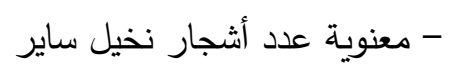
- معنوبة عدد أشجار تخيل خضراوي عندي - معنوية عدد أشجار نخيل حلاوي أنجار نحيل خفراي 
- معنوية عدد أشجار نخيل ديري

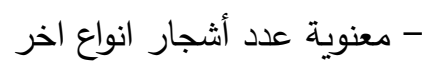

منهجية البحث:

استخدمنا في هذا البحث البيانات التي تم الحصول عليها من:

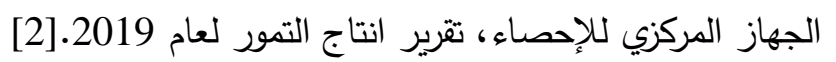

تم استعمال حقيبة البرامج الإحصائية الجاهزة (SPSS) في تحليل البيانات واستخراج النتائج، وقد تمرئ

استعمال التحليل الاحصائي متعدد المتغيرات (أنموذج الانحدار الخطي المتعدد) لتحليل العلاقة بين المتغيرات

والوصول إلى المتغيرات التي تؤثر فعلا على انتاج التمور لعام 2019.

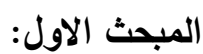

لقد وقع اختيارنا على تمور النخيل وهو المحصول الاقتصادي المهم من هذه الثجرة والذي يكون ذا قيمة

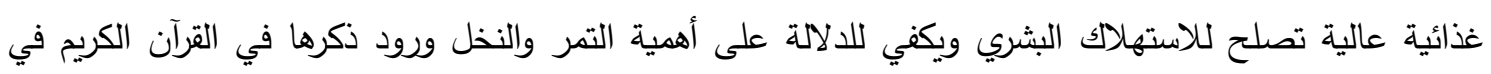
مواضع متعددة منها قوله تعالى: "وزروع ونخل طلعها هضيم" سورة الثعراء الآية (148)، وقوله: :فيها فاكهة ونخل فئل

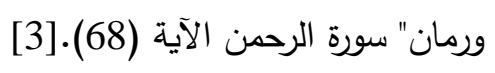

كما ورد في الحديث أن رسول الله صلى الله عليه واله وسلم قال: "أكرموا عمتتا النخلة فإنها خلقت من

$$
\text { الطين الذي خلق منه آدم عليه السلام". }
$$

وتحتوي التمور على قيمة غذائية عالية وتعتبر كقوت أساسي للإنسان منذ القدم ويعتبر ثمار التمور أعلى لقدي

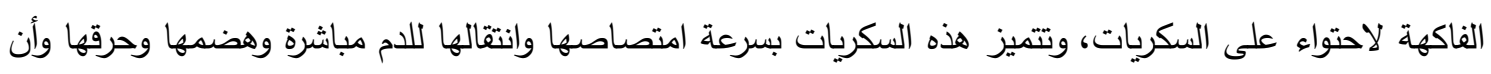
10 حبات تمر (حوالي 100 جرام) يوميا تغني الإنسان بكامل احتياجاته اليومية من المغنيسيوم والمنغنيز والنحاس ولتساه

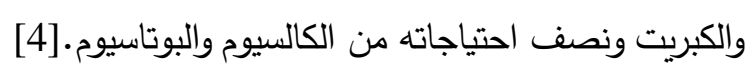

1-1 التمور العراقية.. صراع من أجل البقاء أمام المستورد

ومنذ نهاية الستينيات من القرن الماضي، كان العراق يصدر نحو 75\% من تمور العالم، ويحتل المراتب

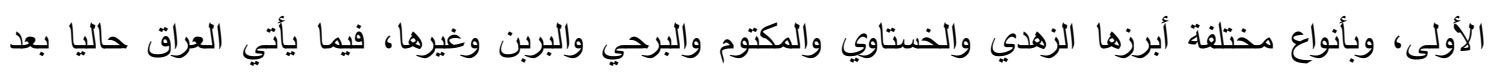

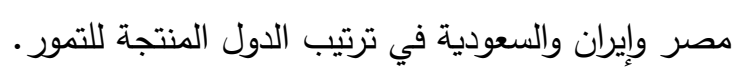

الإهمال الحكومي وقلة الدعم والحروب وسوء التسويق، أبرز أسباب انهيار صناعة التمور التباب العراقية

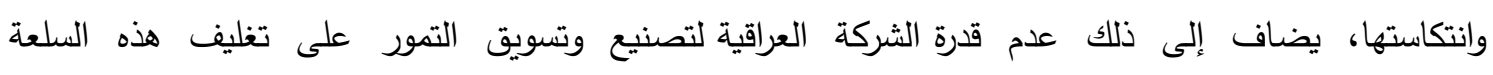

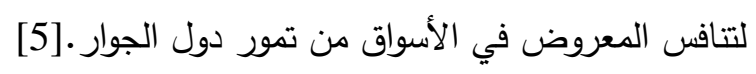

المبحث الثاني:

2-1 الانحدار الخطي المتعدد (Multiple Linear Regression):

في المسائل الإحصائية التطبيقية نضطر إلى تقسير قيم متغير معين وليكن Y بواسطة مجموعة من

المتغيرات ولتكن 
والعلاقة بين المتغير المعتمد $X_{1}, X_{2}, \ldots, X_{m}$

$Y=\beta_{0}+\beta_{1} X_{1}+\beta_{2} X_{2}+\cdots+\beta_{m} X_{m}$

الرياضية لأنموذج الانحدار المتعدد هي كالاتي:

وبإضافة المتغير العشوائي Random Variable) $U$ (إلى الأنموذج أعلاه، فان هذا الأنموذج سيتحول إلى أنموذج

$Y_{i}=\beta_{0}+\sum_{j=1}^{k} \beta_{j} X_{i j}+U_{i}$

احتمالي/عشوائي

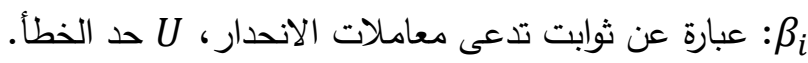
: $\beta_{0}$

$\hat{y}=b_{0}+b_{1} x_{1}+b_{1} x_{2}+\cdots+\beta_{m} x_{m}$ وتقدير الأنموذج باستعمال بيانات العينة بالثكل الآتي:

إذ و

قيم $b_{i}$ تدعى معاملات الانحدار الجزئية (Partial Regression Coefficients)، لذلك فأن

ت7]

2-2 تقدير معلمات الأنموذج (Estimate Parameters of the Model):

احدى طرائق تقدير معالم أنموذج الانحدار هي طريقة المربعات الصغرى الاعتيادية، إن استعمال طريقة المربعات الصغرى (Least Squares Method) في تقدير المعلمات للأنموذج تتميز بأنها تختار أحسن أنموذج مطابق للبيانات بحيث تجعل مجموع مربعات الخطأ اقل ما يمكن فالأنموذج بصورة عامة باستعمال المصفوفات $\hat{\beta}=\left(X^{\prime} X\right)^{-1} X^{\prime} \underline{Y}$

يمكن كتابته بالصيغة الآتية: [8] وان خواص المقدرات بطريقة المربعات الصغرى هي:-

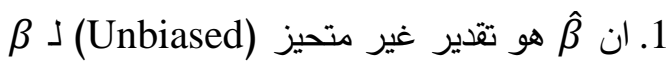

$E(\hat{\beta})=\beta$

2.ان مصفوفة التباين والتباين المشترك لـ مر هو (1) V) ويساوي $\operatorname{var}-\operatorname{cov}(\underline{\hat{\beta}})=\sigma^{2} I_{n}\left(X^{\prime} X\right)^{-1}=\sigma^{2}\left(X^{\prime} X\right)^{-1}$

3. يكون تباين المعلمات المقدرة هو اقل ما يمكن (Minimum Variance).

2-3

جودة الملاءمة تعني كم إن الأنموذج الإحصائي هو ملائم لتوزيع بيانات عينة الدراسة، فمقاييس جودة الملاءمة تقيس التقارب بين القيم المشاهدة والمتوقعة للأنموذج.

اختبار F يعني مدى اقتراب القيم المشاهدة من خط التقدير ، إذ إن مدى التوافق بين الجزء المشاهد والجزء لهنه

المتوقع يزودنا بدليل الملاعمة او عدم الملاءمة للأنموذج لاختبار الفرضية الاتية:

$H_{0}: \beta_{1}=\beta_{2}=\beta_{3}=\cdots=\beta_{q}=0 \quad$ v.s

$H_{1}$ : not all $\beta_{i}(i>0)$ are equal to zero 
وهو احد الاختبارات المهمة المستخدمة لملاءمة جودة الأنموذج او تحديده ويحسب بقسمة متوسط مربعات

الانحدار على متوسط مربعات الخطأ وكما في الصيغة الأتية:[10

$F=\frac{\left(\dot{b} \dot{X} Y-n \bar{Y}^{2}\right) / k}{(\overline{\bar{Y}} Y-\bar{b} \dot{X} Y) /(n-k-1)}$

وبقسمة كل من البسط والمقام على مجموع مربعات الانحرافات الكلية

$F=\frac{R^{2} / k}{\left(1-R^{2}\right) /(n-k-1)}$

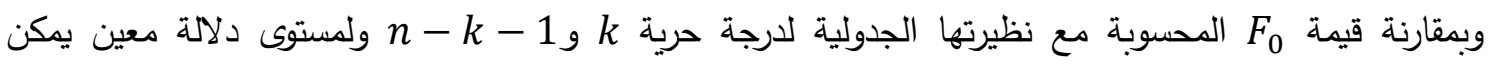
التعرف على مدى معنوية العلاقة الخطية المقترحة او لاختبار مدى تأثير المتغيرات المستقلة

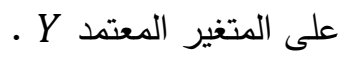

اذا كانت $F_{0}$ المحتسبة اقل من F الجدولية نقبل فرضية العدم H $_{0}$ اي ان المتغيرات المستقلة مجتمعة ليس لها تأثير معنوي على الانحدار اما في الحالة التي تكون فيها Fo المحتسبة اكبر من F الجدولية يؤخذ بالفرضية البديلة $H_{1}$ والقائلة بان المتغيرات المستقلة مجتمعة لها تأثير معنوي على الانحدار .

\section{2-4 معامل التحديد (The Coefficient of Determination) :}

يمثل نسبة الانحرافات الكلية المشروحة بواسطة معادلة الانحدار التقديرية او نسبة مساهمة معادلة الانحدار

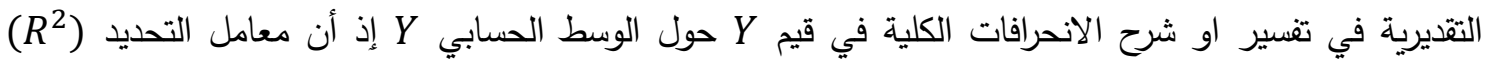

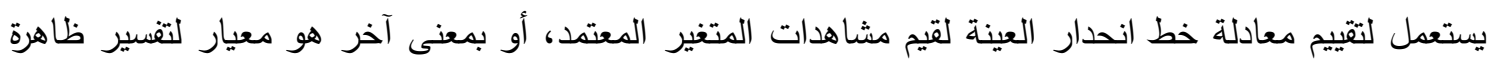
معينة. ويمكن ان يعبر عن معامل التحديد بانه النسبة بين الانحرافات الموضحة الى الانحرافات الكلية اي ان ان النيل $R^{2}=\frac{S S T-S S E}{S S T}=1-\frac{S S E}{S S T}$ $0 \leq R^{2} \leq 1$

وعند تقدير معادلات أنموذج الانحدار يكون من الضروري استعمال معامل التحديد ${ }^{2}$ لمعرفة مدى جودة توفيق خط الانحدار المقدر لكل من المتغير التابع والمتغير المستقل. ويمتاز معامل التحديد بأنه لو أُضيف متغير مستقل إلى الأنموذج فإن مجموع مربعات الخطأ (البواقي)

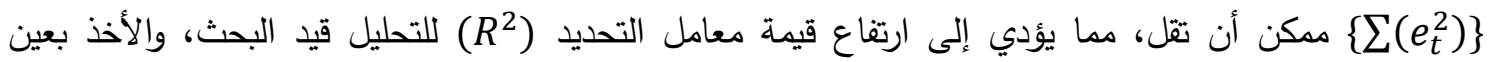
Adjusted Coefficient of ) الاعتبار عدد المتغيرات الجديدة كلها في حساب معامل التحديد المعدل Determination

$\operatorname{adj} R^{2}=1-\left[\left(1-R^{2}\right) \frac{n-1}{n-k-2}\right]$

إن القيمة المثلى لمعامل التحديد تختلف تبعاً لنوع الدراسة، ففي التطبيقات البيولوجية مثلا فأن نسبة 60\%

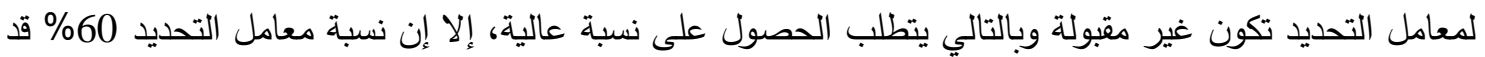
تكون مقبولة في دراسات أخرى وعندما يصعب الحصول على معامل تحديد أعلى من تلك النسبة، مع ملاحظة إلى [13] شرط معنوية الانحدار يوجد أكثر من نوع من نماذج الانحدار كل منها يمثل طريقة معينة لمعالجة مشكلة الانحدار المتعدد. ولتأكيد نتائج الدراسة استخدمنا نوعين من نماذج الانحدار

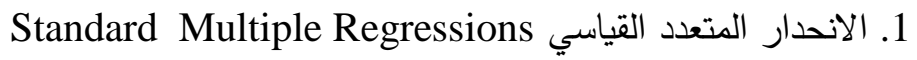


في هذا النوع ندخل كل المتغيرات المستقلة دفعة واحده دون فحص المتغيرات المستقلة وأثرها المعنوي على

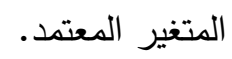

2. الانحدار المتعدد التدريجي Stepwise Multiple Regressions في هذا النوع يتم ادخال المتغيرات المستقلة على خطوات حسب قوة ارتباطها بالمتغير التابع.

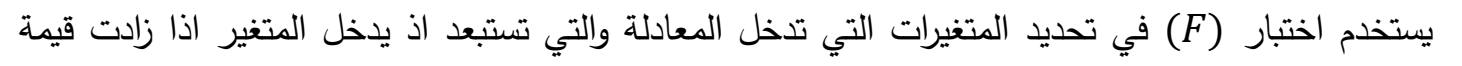

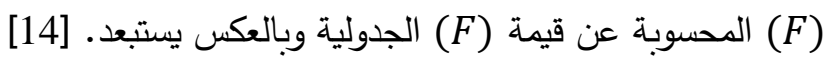
المبحث الثالث: الجانب التطبيقي: - 20 - n 3-1 العلاقة بين كمية انتاج التمور لعام 2019 وعدد اشجار النخيل لعام 2019 حسب الانواع وصف البيانات جدول (1) يمثل وصف المتغيرات.

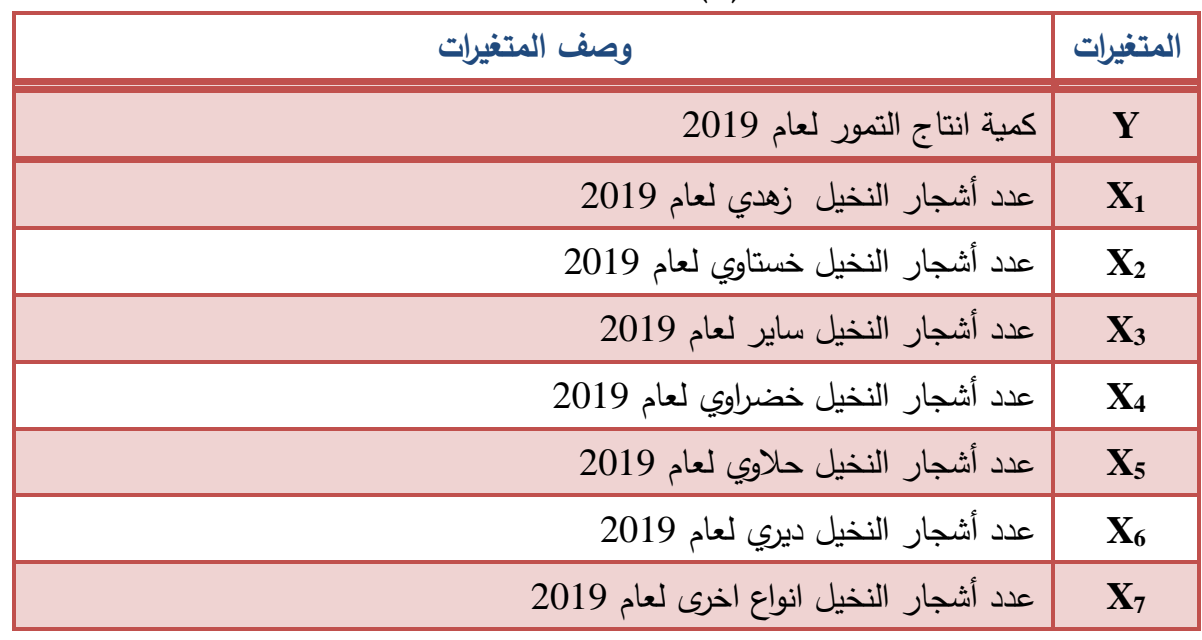

3-2 النتائج والمناقشة (طريقة الانحدار الخطي المتعدد):-

بتطبيق الانحدار المتعدد الخطي القياسي باستخدام برنامج Spss في دراسة العلاقة بين المتغير التابع

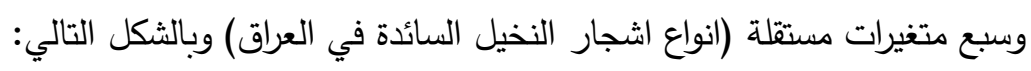
جدول (2) يوضح الاحصاءات الوصفية لمتغيرات الدراسة

\begin{tabular}{|lccc|}
\hline & \multicolumn{3}{c|}{ Descriptive Statistics } \\
\hline & Mean & Std. Deviation & N \\
\hline $\mathrm{y}$ & 53136.18 & 36103.949 & 11 \\
\hline $\mathrm{x} 1$ & 27124.00 & 24321.928 & 11 \\
\hline $\mathrm{x} 2$ & 5688.00 & 4790.382 & 11 \\
\hline x3 & 2458.73 & 2535.019 & 11 \\
\hline x5 & 1347.64 & 2746.739 & 11 \\
\hline x6 & 2440.55 & 2370.371 & 11 \\
\hline x7 & 11216.55 & 14074.420 & 11 \\
\hline
\end{tabular}

المصدر: نتائج ياستخدام بيانات الدراسة بالطريقة الانحدار الخطي القياسي. 
من جدول (2) يتضح أن متوسط انتاج التمور لعام 2019 هو (53136.18) بانحراف معياري (36103.949) واعلي متوسط كان لأشجار نخيل الزهدي (27124.00) بانحراف معياري (24321.928) واقل متوسط كان لأثجار البرحي (1347.64) بانحراف معياري (2746.739) ثم اشجار نخيل الديري (2440.55) بانحراف معياري (2370.371) واشجار نخيل الساير (2458.73) بانحراف معياري (4790.382) على الترتيب.

\section{جدول (3) يوضح القوة التقسيرية للنموذج}

\begin{tabular}{|c|c|c|c|c|}
\hline \multicolumn{5}{|c|}{ Model Summary ${ }^{b}$} \\
\hline Model & $\mathrm{R}$ & R Square & $\begin{array}{l}\text { Adjusted R } \\
\text { Square }\end{array}$ & Std. Error of the Estimate \\
\hline 1 & .954 & .998 & .955 & 2584.301 \\
\hline \multicolumn{5}{|c|}{ a. Predictors: (Constant), x7, x6, x5, x1, x3, x2 } \\
\hline b. Depe & ariab & & & \\
\hline
\end{tabular}

المصدر: نتائج spss باستخدام بيانات الدراسة بالطريقة الانحدار الخطي القياسي.

يتضح من جدول (3) ان المتغيرات المستقلة فسرت (955.) من التغيرات الحاصلة في المتغير المعتمد

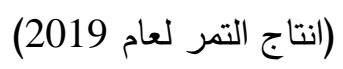

جدول (4) يوضح نتائج تحليل التباين لاختبار معنوية الانحدار

\begin{tabular}{|c|c|c|c|c|c|c|}
\hline \multicolumn{7}{|c|}{ ANOVA $^{\mathbf{a}}$} \\
\hline & Model & Sum of Squares & df & Mean Square & $\mathrm{F}$ & Sig. \\
\hline \multirow{3}{*}{1} & Regression & 13008237237.440 & 6 & 2168039539.573 & 324.624 & $.000^{\mathrm{b}}$ \\
\hline & Residual & 26714440.197 & 4 & 6678610.049 & & \\
\hline & Total & 13034951677.636 & 10 & & & \\
\hline \multicolumn{7}{|c|}{ a. Dependent Variable: y } \\
\hline \multicolumn{7}{|c|}{ b. Predictors: (Constant), x7, x6, x5, x1, x3, x2 } \\
\hline
\end{tabular}

المصدر : نتائج spss باستخدام بيانات الدراسة بالطريقة الانحدار الخطي القياسي.

يتضح من جدول (4) رفض الفرضية الصفرية التي تتص بان الانحدار بين المتغير التابع والمتغيرات

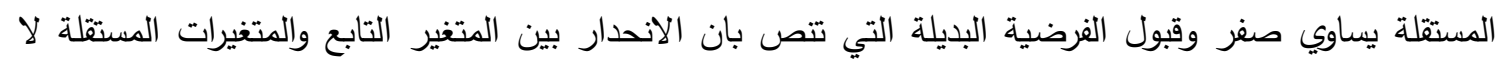
يساوي صفر اي ان الانحدار معنوي بين المتغير المعتمد والمتغيرات المستقلة بمعنى اخر توجد علاقة بين متغيرات الدراسة.

\begin{tabular}{|c|c|c|c|c|c|c|}
\hline & \multicolumn{6}{|c|}{ جدول (5) يوضح تفصيل معاملات معادلة الانحدار } \\
\hline & \multirow[t]{2}{*}{ Model } & \multicolumn{2}{|c|}{$\begin{array}{l}\text { Unstandardized } \\
\text { Coefficients }\end{array}$} & \multirow{2}{*}{$\begin{array}{c}\text { Standardized } \\
\text { Coefficients } \\
\text { Beta }\end{array}$} & \multirow[t]{2}{*}{$\mathrm{T}$} & \multirow[t]{2}{*}{ Sig. } \\
\hline & & B & Std. Error & & & \\
\hline \multirow{7}{*}{1} & (Constant) & -311.229 & 2058.067 & & $-.151-$ & .887 \\
\hline & $\mathrm{x} 1$ & 1.037 & .106 & .699 & 9.795 & .001 \\
\hline & $\mathrm{x} 2$ & .763 & .606 & .101 & 1.260 & .276 \\
\hline & $\mathrm{x} 3$ & 2.465 & .635 & .173 & 3.881 & .018 \\
\hline & $\mathrm{x} 5$ & .251 & .463 & .019 & .542 & .616 \\
\hline & $\mathrm{x} 6$ & 1.123 & .567 & .074 & 1.981 & .119 \\
\hline & $\mathrm{x} 7$ & 1.055 & .089 & .411 & 11.809 & .000 \\
\hline
\end{tabular}

المصدر : نتائج spss باستخدام بيانات الدراسة بالطريقة الانحدار الخطي القياسي. 
يتضح من جدول (5) أكثر المتغيرات تأثيرا على متغير الانتاج وبشكل دال احصائيا أشجار النخيل انواع

اخرى ثم اشجار نخيل الزهدي ثم اشجار نخيل الساير ولم تظهر المتغيرات المستقلة الاخرى أي دلالة احصائية بمعنى اخر ان أشجار نخيل البرحي والديري والخضراوي والخستاوي لم يكن لها تأثير على انتاج التمور لعام 2019 رغم انها تعتبر من اصناف الدرجة الاولى.

Histogram

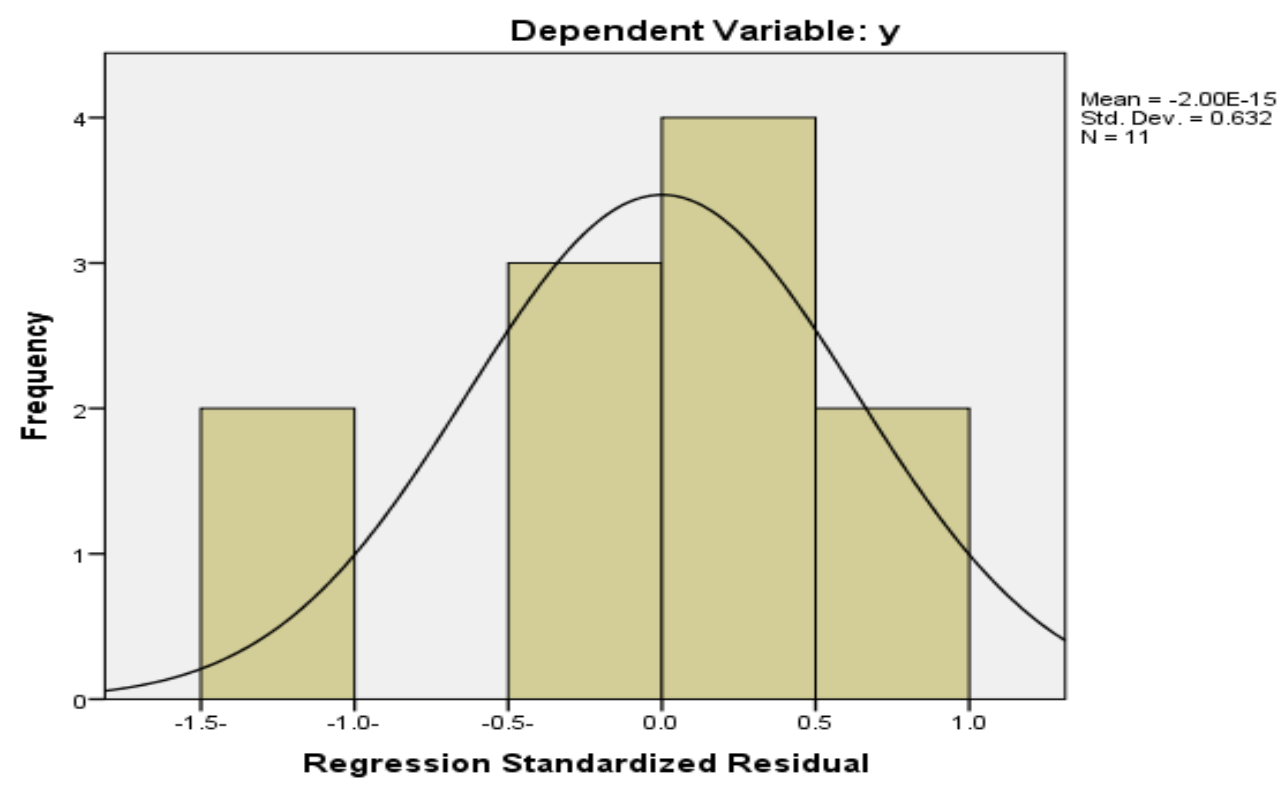

شكل (1) المدرج التكراري للبيانات الدراسة

يتضح من رسم المدرج التكراري ان البيانات تتبع التوزيع الطبيعي وهو شرط من شروط الانحدار . Normal P-P Plot of Regression Standardized Residual

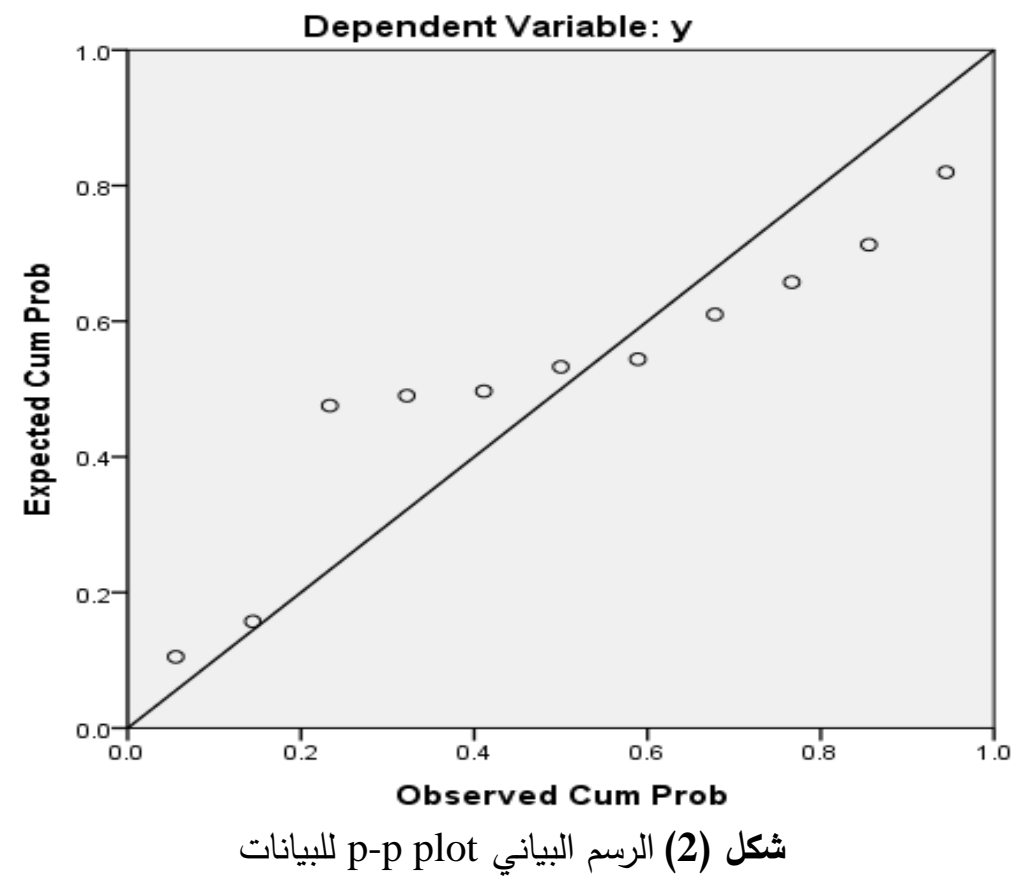

يوضح الرسم البياني p-p plot أن البيانات تتجمع حول الخط المستقيم وبالتالي فان البواقي تتوزع حسب التوزيع الطبيعي وهو شرط اخر من شروط الانحدار . لريح 


\section{3-3 النتائج والمناقشة (طريقة الانحدار الخطي المتعدد التدريجي):-}

سيتم اجراء الانحدار الخطي المتعدد بطريقة الانحدار الخطي التدريجي لتأكيد نتائج الدراسة.

جدول (6) المتغيرات التي ادخلت في معادلة انحدار وطريقة استبعاد المتغيرات الاخرى.

\begin{tabular}{|c|c|c|l|}
\hline \multicolumn{2}{|c|}{ Variables Entered/Removed } \\
\hline Model & $\begin{array}{c}\text { Variables } \\
\text { Entered }\end{array}$ & $\begin{array}{c}\text { Variables } \\
\text { Removed }\end{array}$ & \multicolumn{1}{c|}{ Method } \\
\hline 1 & $\mathrm{X} 7$ & $\cdot$ & $\begin{array}{l}\text { Stepwise (Criteria: Probability-of-F-to-enter }<= \\
\text {.050, Probability-of-F-to-remove }>=.100) .\end{array}$ \\
\hline 2 & $\mathrm{X} 1$ & $\cdot$ & $\begin{array}{l}\text { Stepwise (Criteria: Probability-of-F-to-enter }<= \\
\text {.050, Probability-of-F-to-remove }>=.100) .\end{array}$ \\
\hline 3 & $\mathrm{X} 3$ & $\cdot$ & $\begin{array}{l}\text { Stepwise (Criteria: Probability-of-F-to-enter }<= \\
\text {.050, Probability-of-F-to-remove }>=.100) .\end{array}$ \\
\hline
\end{tabular}

المصدر : نتائج spss باستخدام بيانات الدراسة بالطريقة الانحدار الخطي التدريجي.

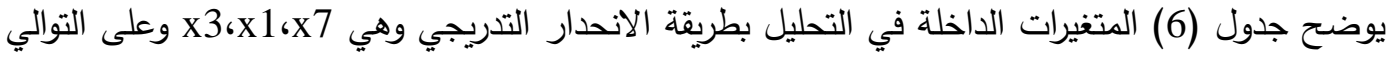

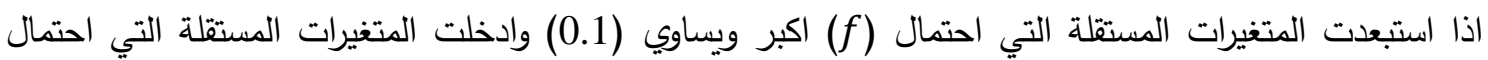
(0.05) (صغر ويساوي (f)

جدول (7) يوضح القوى التقسيرية للنماذج

\begin{tabular}{|c|c|c|c|c|}
\hline \multicolumn{5}{|c|}{ Model Summary $^{\mathbf{e}}$} \\
\hline Model & $\mathrm{R}$ & R Square & Adjusted R Square & Std. Error of the Estimate \\
\hline 1 & $.932^{\mathrm{a}}$ & .868 & .854 & 13813.971 \\
\hline 2 & $.969^{\mathrm{b}}$ & .938 & .923 & 10043.879 \\
\hline 3 & $.983^{\mathrm{c}}$ & .967 & .953 & 7820.706 \\
\hline
\end{tabular}

المصدر : نتائج spss باستخدام بيانات الدراسة بالطريقة الانحدار الخطي التدريجي.

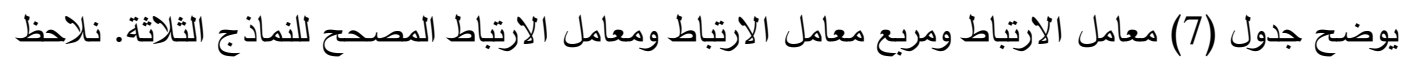
أعلاه قيمة لمعامل الارتباط المصحح في النموذج الثالث. سيتم الاعتماد على النموذج الثالث حيث تفسر متغيرات

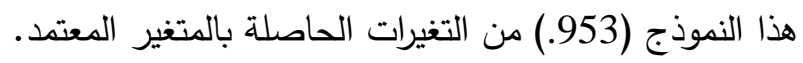
جدول (8) يوضح نتائج تحليل التباين لاختبار معنوية الانحدار

\begin{tabular}{|c|c|c|c|c|c|c|}
\hline \multicolumn{7}{|c|}{ ANOVA $^{a}$} \\
\hline & Model & Sum of Squares & df & Mean Square & $\mathrm{F}$ & Sig. \\
\hline \multirow{3}{*}{1} & Regression & 11317519460.534 & 1 & 11317519460.534 & 59.308 & $.000^{\mathrm{b}}$ \\
\hline & Residual & 1717432217.103 & 9 & 190825801.900 & & \\
\hline & Total & 13034951677.636 & 10 & & & \\
\hline \multirow{3}{*}{2} & Regression & 12227915598.528 & 2 & 6113957799.264 & 60.607 & $.000^{c}$ \\
\hline & Residual & 807036079.108 & 8 & 100879509.889 & & \\
\hline & Total & 13034951677.636 & 10 & & & \\
\hline \multirow{3}{*}{3} & Regression & 12606807530.572 & 3 & 4202269176.857 & 68.706 & $.000^{\mathrm{d}}$ \\
\hline & Residual & 428144147.064 & 7 & 61163449.581 & & \\
\hline & Total & 13034951677.636 & 10 & & & \\
\hline
\end{tabular}

المصدر : نتائج spss باستخدام بيانات الدراسة بالطريقة الانحدار الخطي التدريجي .

يتضح من جدول (8) نتائج تحليل التباين لاختبار معنوبة الانحدار إذا نلاحظ ان مستوى المعنوية القل من 0.05 للنماذج الثلاث وبالتالي رفض الفرضية الصفرية التي تنص بان الانحدار بين المتغير التابع والمتغيرات 
المستقلة يساوي صفر وقبول الفرضية البديلة التي تتص بان الانحدار بين المتغير التابع والمتغيرات المستقلة لا يساوي صفر اي ان الانحدار معنوي بين المتغير المعتمد والمتغيرات المستقلة بمعنى اخر توجد علاقة بين متغيرات الدراسة.

جدول (9) يوضح تفصيل معاملات معادلة الانحدار

\begin{tabular}{|c|c|c|c|c|c|c|c|c|c|}
\hline \multicolumn{10}{|c|}{ Coefficients $^{\mathrm{a}}$} \\
\hline & \multirow{2}{*}{ Model } & \multicolumn{2}{|c|}{$\begin{array}{l}\text { Unstandardized } \\
\text { Coefficients }\end{array}$} & \multirow{2}{*}{$\begin{array}{c}\text { Standardized } \\
\text { Coefficients } \\
\text { Beta }\end{array}$} & \multirow{2}{*}{$\mathrm{t}$} & \multirow{2}{*}{ Sig. } & \multicolumn{3}{|c|}{ Correlations } \\
\hline & & B & Std. Error & & & & $\begin{array}{l}\text { Zero- } \\
\text { order }\end{array}$ & Partial & Part \\
\hline \multirow{2}{*}{1} & (Constant) & 13190.943 & 6652.198 & & 1.983 & .079 & & & \\
\hline & X7 & 7.023 & .912 & .932 & 7.701 & .000 & .932 & .932 & .932 \\
\hline \multirow{3}{*}{2} & (Constant) & 13087.596 & 4836.811 & & 2.706 & .027 & & & \\
\hline & $\mathrm{X} 7$ & 5.252 & .887 & .697 & 5.921 & .000 & .932 & .902 & .521 \\
\hline & $\mathrm{X} 1$ & .907 & .302 & .354 & 3.004 & .017 & .817 & .728 & .264 \\
\hline \multirow{4}{*}{3} & (Constant) & 12359.404 & 3777.549 & & 3.272 & .014 & & & \\
\hline & $\mathrm{X} 7$ & 2.522 & 1.296 & .335 & 1.945 & .001 & .932 & .592 & .133 \\
\hline & $\mathrm{X} 1$ & .972 & .237 & .379 & 4.110 & .005 & .817 & .841 & .282 \\
\hline & $\mathrm{X} 3$ & .572 & .230 & .386 & 2.489 & .042 & .897 & .685 & .170 \\
\hline
\end{tabular}

المصدر : نتائج spss باستخدام بيانات الدراسة بالطريقة الانحدار الخطي التدريجي.

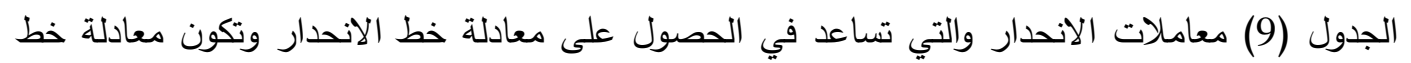

الانحدار وحسب النموذج الثالث لان هذا النموذج أفضل نموذج يفسر التغيرات الحاصلة بالمتغير المعتمد

Predicted $\mathrm{Y}=12359.404+.972 \mathrm{X} 1+.572 \mathrm{X} 3+2.522 \mathrm{X} 7$

جدول (10)

\begin{tabular}{|c|c|c|c|c|c|c|}
\hline \multicolumn{7}{|c|}{ Excluded Variables $^{\mathbf{a}}$} \\
\hline \multirow{2}{*}{\multicolumn{2}{|c|}{ Model }} & \multirow{3}{*}{$\begin{array}{c}\text { Beta In } \\
315^{\mathrm{b}}\end{array}$} & \multirow{3}{*}{$\frac{\mathrm{t}}{1.184}$} & \multirow{3}{*}{$\begin{array}{l}\text { Sig. } \\
.270\end{array}$} & \multirow{3}{*}{$\begin{array}{c}\begin{array}{c}\text { Partial } \\
\text { Correlation }\end{array} \\
.386\end{array}$} & \multirow{3}{*}{$\begin{array}{c}\text { Collinearity Statistics } \\
\text { Tolerance } \\
.198\end{array}$} \\
\hline & & & & & & \\
\hline \multirow{6}{*}{1} & $\begin{array}{l}\mathrm{x} 1 \\
\mathrm{x} 2\end{array}$ & & & & & \\
\hline & $\mathrm{x} 3$ & $.069^{\mathrm{b}}$ & .502 & .629 & .175 & .842 \\
\hline & $\mathrm{x} 4$ & $.170^{\mathrm{b}}$ & 1.436 & .189 & .453 & .936 \\
\hline & $\times 5$ & $-.024-^{\mathrm{b}}$ & $-.182-$ & .860 & $-.064-$ & .957 \\
\hline & & $.354^{\mathrm{b}}$ & 3.004 & .017 & .728 & .559 \\
\hline & x6 & $-.064-^{\mathrm{b}}$ & $-.508-$ & .625 & $-.177-$ & .997 \\
\hline \multirow{5}{*}{2} & $\mathrm{X} 2$ & $.386^{\mathrm{c}}$ & 2.489 & .042 & .685 & .195 \\
\hline & $\mathrm{x} 3$ & $.053^{\mathrm{c}}$ & .530 & .613 & .196 & .840 \\
\hline & $\mathrm{x} 4$ & $.112^{\mathrm{c}}$ & 1.237 & .256 & .424 & .884 \\
\hline & $\mathrm{x} 5$ & $-.011-^{\mathrm{c}}$ & $-.114-$ & .913 & $-.043-$ & .955 \\
\hline & x6 & $-.005-^{c}$ & $-.052-$ & .960 & $-.020-$ & .946 \\
\hline \multirow{4}{*}{3} & $\mathrm{X} 2$ & $.221^{\mathrm{d}}$ & 6.444 & .071 & .935 & .589 \\
\hline & $\mathrm{x} 4$ & $.157^{\mathrm{d}}$ & 3.218 & .0178 & .796 & .846 \\
\hline & $x 5$ & $.096^{\mathrm{d}}$ & 1.250 & .258 & .455 & .730 \\
\hline & $\mathrm{x} 6$ & $.154^{\mathrm{d}}$ & 2.180 & .072 & .665 & .610 \\
\hline
\end{tabular}

المصدر : نتائج spss باستخدام بيانات الدراسة بالطريقة الانحدار الخطي التدريجي.

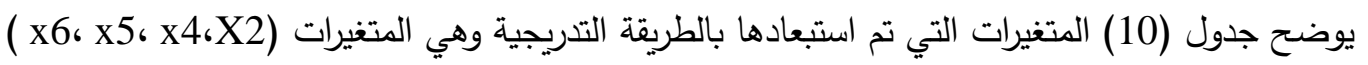

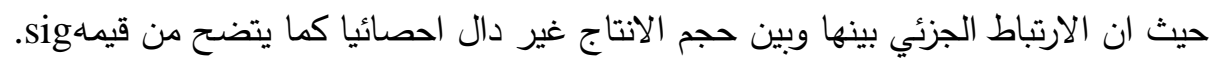


الاستنتاجات:

من الجانب التطبيقي وتحليل البيانات التي تم جمعها من عينة البحث تم التوصل الى مجموعة من

الاستتاجات كان من اهمها

1. المتغيرات المستقلة السبعة تقسر الهن 0.95 من المتغير المعتمد.

2. ان المتغيرات المستقلة المستعملة في البحث لم تفسر جميع التباين الموجود في المتغير المعتمدة بمعنى أن هنالك متغيرات أخرى قد يكون لها تأثير معنوي لكن لم تستعمل بالبحث.

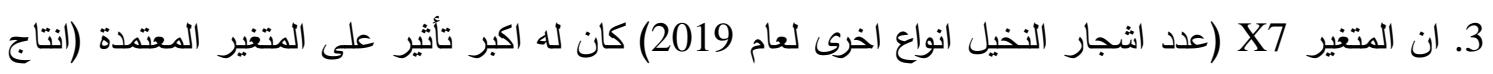

$$
\text { التمور لعام 2019) بمستوى معنويه يساوي (200) (200). (2019). }
$$

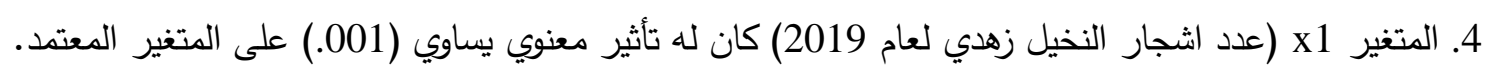

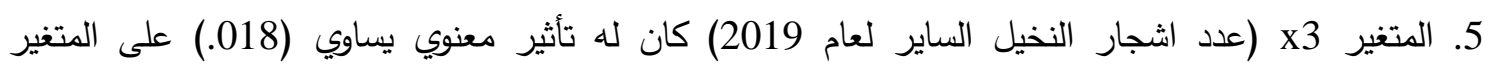
المعتمد.

6. المتغيرات المستقلة الاخرى المتمثلة اشجار نخيل الحلاوي والخستاوي والخضراوي والديري لم تظهر معنويه في نموذج الانحدار رغم ان تلك الاصناف تعتبر اصناف الدرجة الاولى للتصدير.

التوصيات:

1. ان سياسة انتاج التمور في العراق تحتاج الى مراجعة شاملة من قبل الجهات ذات العلاقة والمتمثلة في مجلس

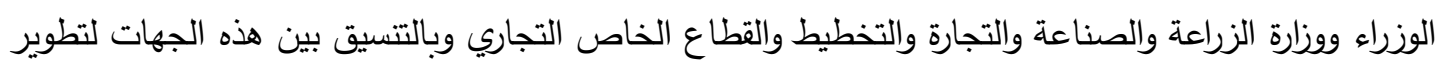

$$
\text { وزيادة الانتاج وتحقيق منافسه في السوق العالمية. }
$$

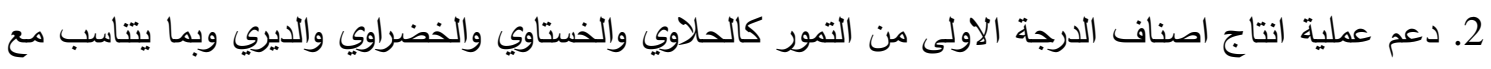
حجم الطلب على هذا المنتجات الوطنية الذي من الممكن ان تساهم في تتوع هيكل الصادرات وايجاد اسواق

$$
\text { مناسبة لتصريف الفائض من التمور الى الاسواق العربية. }
$$

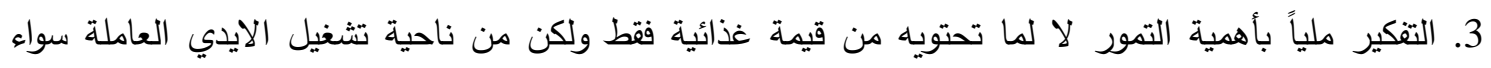

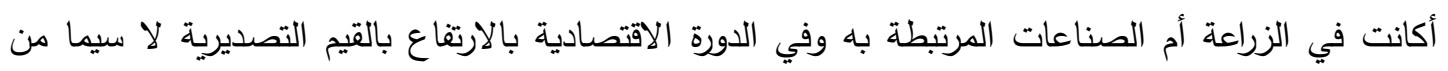
الانواع النادرة المخصوصة بالعراق تحديداً.

4. الاهتمام بتوفير مستلزمات مكافحة افات وحشرات النخيل مجانا او بأسعار رمزية لأصحاب بساتين النخيل. 


$$
\begin{aligned}
& \text { المصادر } \\
& \text { بحسب ورودها بالبحث } \\
& \text { مريم جلال العتاب، "تحليل إحصائي لإنتاج التمور في محافظات العراق للمدة 2013-2002"، بحث دبلوم، }
\end{aligned}
$$

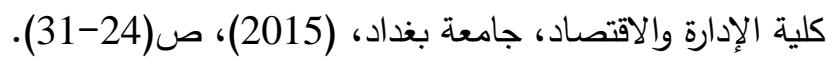

$$
\begin{aligned}
& \text { الجهاز المركزي للإحصاء، تقرير أنتاج التمور لعام } 2019 . \\
& \text { كاظم، أموري هادي ومسلم، باسم شليبه، (2002) "القياس الاقتصادي المتقدم النظرية والتطبيق"، مطبعة }
\end{aligned}
$$

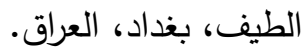

$$
\begin{aligned}
& \text { كاظم، أموري هادي وعيفان، محمد مناجد، (1988)، "مقدمة في تحليل الانحدار الخطي"، كلية الادارة }
\end{aligned}
$$

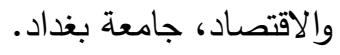

$$
\begin{aligned}
& \text { شابا، صلاح فهمي، "التحليل الاقتصادي والقياسي لدوال الإنتاج والتكاليف لمحصول الزيتون في ناحية } \\
& \text { بعشيقة بمحافظة نينوى للموسم الزراعي (2010) "، رسالة ماجستير، كلية الزراعة، جامعة بغداد (2012). } \\
& \text { عباس، علي خضير، "استخدام أنموذج الانحدار اللوجستي في التتبؤ بالدوال ذات المتغيرات الاقتصادية } \\
& \text { التابعة النوعية"، مجلة كركوك للعلوم الادارية والاقتصادية، مجلد 2، العدد 2، 2012. } \\
& \text { صالح، طارق عزيز، 2009، "مقارنة بعض الطرائق الحصينة في تحليل الارتباط القويم الخطي باستخدام }
\end{aligned}
$$

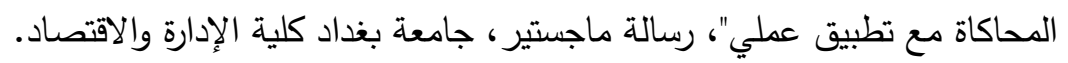

$$
\begin{aligned}
& \text { جودة، محفوظ، "التحليل الإحصائي المتقدم باستخدام (spss)"، عمان (2008). } \\
& \text { عبد الله، سهيلة نجم، 2008، "استخدام تحليل الارتباط القويم لدراسة تأثير مجموعة من العوامل على انتاج } \\
& \text { المحاصيل الاستراتيجية"، مجلة الادارة والاقتصاد، العدد } 73 .
\end{aligned}
$$

[10] https://www.youtube.com/watch?v=R9CnF8p6Zys

[11] Brostrom, C., (1985), "Practical Aspects on The Estimation of The Parameters in Coal's Model for martial Fertility", Demography, Vol. (22), No. (4), PP: 611-637.

[12] Cerone, P., (1996), "On The Effect of The Generalized Renewal Integral Equation Model of Population Dynamic", Genus, Vol. LII-n, 1-2, PP: 53-70.

[13] Goldstient, J., (2002), "Population Momentum for Gradual Demographic Transitions: An Alternative Approach", Demography, Vol. (39), No. (1), PP: 6573.

[14] https://www.alghamdi-biostatistics.com/multipleregression.htm 
بيانات الدراسة

\begin{tabular}{|r|r||r|r|r||r|r||r||}
\hline \multicolumn{1}{|c||}{$\mathbf{y}$} & $\mathrm{x} 1$ & $\mathrm{x} 2$ & $\mathrm{x} 3$ & $\mathrm{x} 4$ & $\mathrm{x} 5$ & $\mathrm{x} 6$ & $\mathrm{x} 7$ \\
\hline 50293 & 22640 & 7875 & 3437 & 1920 & 476 & 5981 & 7964 \\
\hline 135212 & 64057 & 14777 & 544 & 2247 & 396 & 621 & 52570 \\
\hline 93603 & 61710 & 13308 & 1268 & 2357 & 235 & 4507 & 10220 \\
\hline 80947 & 63361 & 8479 & 139 & 644 & 100 & 640 & 7584 \\
\hline 33797 & 22537 & 3812 & 212 & 1159 & 723 & 287 & 5067 \\
\hline 29376 & 19729 & 3995 & 781 & 1529 & 427 & 279 & 2636 \\
\hline 38899 & 22192 & 1872 & 2760 & 1151 & 430 & 301 & 10193 \\
\hline 36054 & 10666 & 2623 & 1972 & 2762 & 1530 & 5310 & 11191 \\
\hline 44270 & 7611 & 1731 & 6952 & 12616 & 834 & 4875 & 9651 \\
\hline 8668 & 1224 & 682 & 1644 & 2018 & 132 & 534 & 2434 \\
\hline 33379 & 2637 & 3414 & 7337 & 3067 & 9541 & 3511 & 3872 \\
\hline
\end{tabular}

